Conclusions: In this study, $78.5 \%$ of patients in east China didn't have goutrelated knowledge. Patients' knowledge on gout is a significant independent determinant of adherence to ULT.

Acknowledgements: This study was supported by grants from the Cultivative Distinguished Young Scholars Project of Nantong University (2nd); the 2015 Graduate Innovation Project of Nantong University (YKC15075); and College graduate research and innovation of Jiangsu Province (KYZZ15-0353).

Disclosure of Interest: None declared

DOI: 10.1136/annrheumdis-2017-eular.3071

\section{AB0894 TREATMENT ADHERENCE TO URATE-LOWERING THERAPY IN CHINESE GOUT PATIENTS}

R. Yin ${ }^{1}$, H. Cao ${ }^{1}$, T. Fu ${ }^{1}$, Q. Zhang ${ }^{1}$, L. Zhang ${ }^{1}$, L. Li ${ }^{2}$, Z. Gu ${ }^{1} .{ }^{1}$ Department of Rheumatology, Affiliated Hospital of Nantong University; ${ }^{2}$ School of Nursing, Nantong University, Nantong, China

Background: Gout, which is characterised by deposition of monosodium urate monohydrate (MSU) in synovial fluid and other tissues, is the most common form of inflammatory arthritis in man and older women. In addition to recurrent acute arthritis, subcutaneous tophi and chronic painful arthritis, gout also affects morbidity and premature mortality. Previous studies have reported that effective ULT can decrease sUA levels enough to prevent further crystal formation, dissolve existing urate crystals and eliminate the causative agent, thus reducing the frequency of acute gout attacks and preventing urate nephropathy, uric acid nephrolithiasis, and the deposition of tophi: a common cause of progressive joint damage, deformity and functional impairment, making gout the only chronic arthritis that can be "cured". However, gout patents' adherence rate to ULT is low, ranged from 10 to $46 \%$. Emerging data suggest that poor adherence to long-term ULT use may be an important contributor to the suboptimal outcomes seen in gout and nonadherence may be worse in gout than in any other chronic disease. This suggests that there is a strong need to make a further study on drug adherence and its risk factors.

Objectives: Non-adherence in gout patients using urate-lowering therapy (ULT) may lead to joint destruction and permanent disability. Purpose of this crosssection survey was to explore Chinese gout patients' adherence rates and investigate potential risk factors for medication non-adherence.

Methods: A total of 129 gout patients were recruited from the Affiliated Hospital of Nantong University from August 2015 to September 2016. Patients were asked to complete a standardized self-report questionnaires (Compliance Questionnaire on Rheumatology, Treatment Satisfaction Questionnaire for Medication version II, Health Assessment Questionnaire, Confidence in gout treatment questionnaire, Gout Knowledge Questionnaire, Patient Health Questionnaire-9, Generalized Anxiety Disorder-7, and 36-Item Short Form Health Survey). Data was analyzed by independent sample t-test, rank sum test, chi-square analysis as well as logistic regression modeling.

Results: Based on CQR, 9.6\% of gout patients were adherent to ULT. Adherence was associated with $\mathrm{HAQ}, \mathrm{GKQ}$, treatment satisfaction for medication, confidence in gout treatment and MCS. Other demographic, clinical and psychological characteristics were not related to adherence. Logistic regression models identified $\mathrm{HAQ}, \mathrm{GKQ}$ and $\mathrm{MCS}$ as predictors of medication non-adherence.

Conclusions: In the current study, $90.4 \%$ of gout patients didn't adhere to their ULT prescription. $H A Q, G K Q$, treatment satisfaction for medication, confidence in gout treatment and MCS were relevant to medication adherence, and $\mathrm{HAQ}$, GKQ and MCS were independent predictors of medication non-adherence in patients with gout. These findings could help medical personnel develop useful interventions to improve gout patients' medication adherence and quality of life. Acknowledgements: This study was supported by grants from the Cultivative Distinguished Young Scholars Project of Nantong University (2nd); the 2015 Graduate Innovation Project of Nantong University (YKC15075); and College graduate research and innovation of Jiangsu Province (KYZZ15-0353).

Disclosure of Interest: None declared

DOI: 10.1136/annrheumdis-2017-eular.3075

\section{AB0895 OSTEOPOROSIS RISK FACTORS IN PATIENTS WITH CALCIUM PYROPHOSPHATE CRYSTAL DEPOSITION DISEASE (CPPD)}

S. Vladimirov, M. Eliseev, O. Zhelyabina, A. Smirnov, S. Glukhova. V. A. Nasonova Research Institute of Rheumatology, Moscow, Russian Federation

Background: Osteoporosis (OP) risk factors (RF) in CPPD patients are not sufficiently studied, although some of these RFs may be more prevalent in CPPD pts than in general population [1]

Objectives: To identify the input of individual RFs into OP development in pts with $C P P D$

Methods: 64 patients with CPPD (35 males and 29 females), but without OP were included into open prospective study. CPPD was confirmed based on McCarty criteria (detection of calcium pyrophosphate crystals in the synovial fluid using polarized light microscopy and detection of chondrocalcinosis based on joint radiography or US examination). 40 pts of those had acute and/or chronic arthritis, and 24 pts had osteoarthritis with CPP crystal depositions. Mean age was $57.6 \pm 10.2$ years, mean follow up $-4.85 \pm 0.96$ years. Bone mass density (BMD) was measured by dual energy X-ray absorptiometry (DXA) at the forearm, lumbar spine and total hip was performed in all pts at baseline. OP was diagnosed by BMD (T-criterion $\leq 2,5$ ). The following OP risk factors were evaluated in this study: sex, age $>55$ years for females and $>65$ years for males, smoking, alcohol, fractures in past medical history, fractures in parents, BMI $\leq 20 \mathrm{~kg} / \mathrm{m}^{2}, \mathrm{BMI} \leq 25 \mathrm{~kg} / \mathrm{m}^{2}$, serum levels of calcium, magnesium, vitamin $\mathrm{D}$, hypoparathyroidism (HPT), chronic kidney disease (GFR $\leq 60 \mathrm{~mL} / \mathrm{min}$ ), intake of diuretics and glucocorticosteroids (GCs), Erythrocyte sedimentation rate (ESR) $>20 \mathrm{~mm} / \mathrm{h}$, C-reactive protein (CRP) $>5,0 \mathrm{mg} / \mathrm{L}$. Odds ratio (OR) $(95 \%$ confidence interval, $\mathrm{Cl}$ ) was estimated for each risk factor and logistic regression analysis was performed. Statistical analysis was made using SPSS v. 11 package, $p$ values of $<0.05$ were considered statistically significant.

Results: OP was identified in 22 (34\%) out of 64 pts with CPPD (13 males and 9 females) by the end of the study. The following factors were associated with OP: age > 55 years in females (odds ratio (OR) $5.0,95 \% \mathrm{Cl} ; 1.021-24,49$; $p=0.047$ ), age $>65$ years in males (OR $3.9,95 \% \mathrm{Cl} 1.3-11.5 ; p=0.014$ ), HPT (OR $15.38,95 \% \mathrm{Cl} 1.7-137.9 ; p=0.015$ ), elevated ESR (OR 3.38, 95\% Cl 1.14-10.0; $p=0.028$ ) and CRP (OR 6.42, 95\% Cl 2.05-20.05; $p=.001$ ) (Fig.1).

Only hypoparathyroidism was identified by logistic regression analysis (sensitivity$71 \%$, specificity $-82 \%$ ) as OP-associated risk factor (OR $14.24,95 \% \mathrm{Cl} 1.05-$ 194.05; $p=0.046$ ) (Table 1).

Table 1. Data from multiple logistic regression analysis

\begin{tabular}{lcccc}
\hline Study & OR & Lower & Upper & $\mathrm{p}$ \\
\hline sex & 1,215 & 0,259 & 5,709 & 0,805 \\
BMI $<25 \mathrm{~kg} / \mathrm{m}^{2}$ & 1,483 & 0,325 & 6,774 & 0,611 \\
Age $>65$ & 1,293 & 0,274 & 6,093 & 0,745 \\
Diuretics intake & 2,771 & 0,189 & 40,617 & 0,457 \\
HPT & 14,245 & 1,046 & 194,055 & 0,046 \\
CKD & 1.025 & 0.082 & 12.876 & 0.985 \\
lower vit.D3 & 0,503 & 0,116 & 2,173 & 0,357 \\
elevated ESR & 3,830 & 0,764 & 19,214 & 0,101 \\
elevated CRP & 3,855 & 0,608 & 24,44 & 0,152 \\
\hline
\end{tabular}

Fig 1. Risk factors for osteoporosis (forest plot)

\section{Odds Ratio $95 \%$ Confidence Interval}

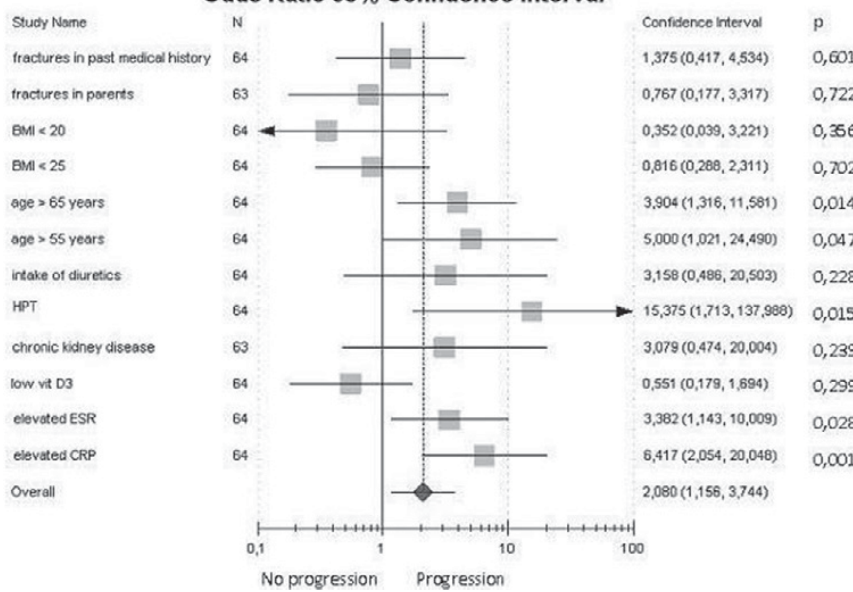

Conclusions: Hypoparathyroidism is the key risk factor for OP in CPPD pts. Among other risk factors chronic inflammation (ESR and CRP levels) is of highest importance.

References:

[1] Ross P.D. Osteoporosis: frequency, consequences, and risk factors. Archives Internal Med.1996; 156:1399-141.

Disclosure of Interest: None declared

DOI: 10.1136/annrheumdis-2017-eular.3755

\section{AB0896 THE EFFECT OF SERUM URIC ACID LEVELS ON TOPHUS STATUS AND FLARES IN PATIENTS WITH GOUT: A SYSTEMATIC REVIEW}

S. Mitchell ${ }^{1}$, L. Manounah ${ }^{1}, \mathrm{H}$. Liedgens ${ }^{2}$, E. Johannes ${ }^{3} .{ }^{1}$ Decision Resources Group, Bicester, United Kingdom; ${ }^{2}$ Head Health Economics and Outcomes Research, Market Access, SBU Europe?; ${ }^{3}$ Head Market Access Europe, Grünenthal $\mathrm{GmbH}$, Aachen, Germany

Background: Gout is a chronic, progressive, inflammatory disease characterised by elevated serum uric acid (sUA) levels. SUA levels above its saturation point result in the deposition of monosodium urate crystals, which lead to gout flares and tophi (1). Multiple studies support the use of sUA levels as a marker for clinical improvements; hence the therapeutic goal is to lower sUA levels $(<6.0 \mathrm{mg} / \mathrm{dL})$ to improve the symptoms of gout and reduce the risk of associated comorbidities $(2,3)$.

Objectives: To conduct a systematic review to identify studies reporting the effect of sUA levels on the incidence of gout flares and tophus status in adult patients with gout/hyperuricaemia, with a focus on publications reporting a correlation between the parameters. 